\title{
BIRKHOFF TYPE DECOMPOSITIONS AND THE BAKER-CAMPBELL-HAUSDORFF RECURSION
}

\author{
KURUSCH EBRAHIMI-FARD, LI GUO, AND DOMINIQUE MANCHON
}

\begin{abstract}
We describe a unification of several apparently unrelated factorizations arisen from quantum field theory, vertex operator algebras, combinatorics and numerical methods in differential equations. The unification is given by a Birkhoff type decomposition that was obtained from the Baker-Campbell-Hausdorff formula in our study of the Hopf algebra approach of Connes and Kreimer to renormalization in perturbative quantum field theory. There we showed that the Birkhoff decomposition of Connes and Kreimer can be obtained from a certain Baker-Campbell-Hausdorff recursion formula in the presence of a Rota-Baxter operator. We will explain how the same decomposition generalizes the factorization of formal exponentials and uniformization for Lie algebras that arose in vertex operator algebra and conformal field theory, and the even-odd decomposition of combinatorial Hopf algebra characters as well as to the Lie algebra polar decomposition as used in the context of the approximation of matrix exponentials in ordinary differential equations.
\end{abstract}

\section{CONTENTS}

1. Introduction 1

2. The general set up 3

2.1. The Baker-Campbell-Hausdorff recursion 3

2.2. Rota-Baxter operator 9

2.3. The case of vanishing weight and the Magnus recursion 13

3. Renormalization in perturbative QFT 16

4. Formal exponentials 18

5. Combinatorial Hopf algebras 20

6. Polar decomposition 23

References 24

\section{INTRODUCTION}

The results presented in this paper grew out of an extension of the study on Rota-Baxter algebras and their applications to areas of mathematics and physics, including quantum field theory, classical integrable systems, number theory, operads, combinatorics and Hopf algebras.

Date: March 14, 2006

2001 PACS Classification: 03.70.+k, 11.10.Gh, 02.10.Hh . 
In recent works of Connes and Kreimer [CK1999, CK2000, CK2001, Krei1999], triggered by Kreimer's seminal paper [Krei1998], new progresses were made in the understanding of the process of renormalization in perturbative quantum field theory, both in terms of its mathematical and its physical contents. These results motivated further studies, among other directions, in the context of Rota-Baxter algebras [EGK2004, EGK2005, EG2005, EGGV2006]. We refer the reader to [EK2005, FGB2005, Krei2002, Man2001] for more details and references in this field.

One key result in the works of Connes and Kreimer is the Birkhoff decomposition of Feynman rules that captures the process of renormalization. Working in a fully algebraic framework of complete filtered Rota-Baxter algebras, it was shown in [EGK2004, EGK2005] that the Connes-Kreimer decomposition follows from an additive decomposition in a Rota-Baxter (Lie) algebra through the exponential map. Thereby the well-known Bogoliubov formulae, which form the backbone of the standard BPHZ renormalization procedure [BoPa1957, Hepp1966, Zim1969], were derived as a special case of a generalization of Spitzer's classical identity. As a side remark we mention here that a similar factorization was independently established as a fundamental theorem for Lie algebras in integrable systems [BBT2003, Sem1983, Sem2000].

These results rely in part on general properties of Rota-Baxter operators, but also on a recursive equation based on the famous Baker-Campbell-Hausdorff formula. We will show that in certain favorable cases we are able to give the recursion in closed form. The main topic of this paper is the exploration of further applications of this recursive equation, which was dubbed the $\mathrm{BCH}$-recursion. We will show its appearance in several fields. First, in the context of Rota-Baxter algebra, it is shown to be a generalization of the Magnus expansion known from matrix initial value problems. Then, by applying the recursion to the decomposition for certain Lie algebras, we derive the factorization of formal exponentials and uniformization in the work of Barron, Huang and Lepowsky [BHL2000] which is itself a generalization of several of their earlier results. Furthermore, we link explicitly the $B C H$-recursion with the even-odd decomposition of characters of connected graded Hopf algebras derived in recent work of Aguiar, Bergeron and Sottile [ABS2003]. This way we achieve an exponential form of their decomposition. This result also relates to our last point. We give a simplified approach to the polar decomposition in the work of Munthe-Kaas and collaborators [MQZ2000, MQZ2001, Zan2004] on numerical solutions of differential equations.

Let us outline the organization of this paper. After the above introduction, Section 2 provides the key result. In subsection 2.1 we introduce a certain Baker-Campbell-Hausdorff type equation as the main object of this work, together with a general factorization theorem for complete filtered associative and Lie algebras. Solutions to this recursion are given under particular assumptions. Subsection 2.2 combines the former results with the notion of RotaBaxter algebra, giving rise to a generalization of Spitzer's classical identity. We relate the $B C H$-recursion to Magnus' expansion in the context of weight zero Rota-Baxter maps. As a motivational example we recall in Section 3 how this, together with Atkinson's factorization theorem, applies to the work of Connes and Kreimer in perturbative quantum field theory. 
Section 4 relates our findings to the work of Barron, Huang and Lepowsky on the factorization of formal exponentials and uniformization. After that, we deduce in Section 5 the even-odd decomposition of combinatorial Hopf algebra characters defined by Aguiar, Bergeron and Sottile and give a closed form for the $B C H$-recursion in this particular setting. Finally, in Section 6 using similar ideas we briefly mention a simplified approach to some results in the work of Munthe-Kaas and collaborators.

\section{The GENERAL SET UP}

In the following $\mathbb{K}$ denotes the base field of characteristic zero, over which all algebraic structures are defined. Many results remain true if it is replaced by a commutative $\mathbb{Q}$-algebra.

Here we establish general results to be applied in later sections. We start with a complete filtered associative algebra $A$ together with a filtration preserving linear map $P$ on $A$ as general setting. We obtain from the Baker-Campbell-Hausdorff $(\mathrm{BCH})$ series a non-linear map $\chi$ on $A$ which we called $B C H$-recursion in [EGK2004, EGK2005, EG2005]. This recursion gives a decomposition on the exponential level, and a one-sided inverse of the BCH series with the later regarded as a map from $A \times A \rightarrow A$. The results naturally apply in the Lie algebra case. We then consider the above setting in the realm of a complete filtered associative Rota-Baxter algebra giving rise to a generalization of Spitzer's classical identity. We conclude this section by showing how the $B C H$-recursion can be seen as a generalization of the Magnus expansion in the context of Rota-Baxter algebras.

2.1. The Baker-Campbell-Hausdorff recursion. Let $A$ be a complete filtered associative algebra. Thus $A$ has a decreasing filtration $\left\{A_{n}\right\}$ of sub-algebras such that $A_{m} A_{n} \subseteq A_{m+n}$ and $A \cong \lim A / A_{n}$ (i.e., $A$ is complete with respect to the topology from $\left\{A_{n}\right\}$ ). For instance, consider for $A$ being an arbitrary associative algebra, the power series $\operatorname{ring} \mathcal{A}:=A[[t]]$ in one (commuting) variable $t$. Another example is given by the subalgebra $\mathcal{M}_{n}^{\ell}(A) \subset \mathcal{M}_{n}(A)$ of strictly (upper) lower triangular matrices in the algebra of $n \times n$ matrices with entries in $A$, and with $n$ finite or infinite. By the completeness of $A$, the functions

$$
\begin{gathered}
\exp : A_{1} \rightarrow 1+A_{1}, \quad \exp (a)=\sum_{n=0}^{\infty} \frac{a^{n}}{n !}, \\
\log : 1+A_{1} \rightarrow A_{1}, \quad \log (1+a)=-\sum_{n=1}^{\infty} \frac{(-a)^{n}}{n}
\end{gathered}
$$

are well-defined and are the inverse of each other.

The Baker-Campbell-Hausdorff formula is the power series $\mathrm{BCH}(x, y)$ in the non-commutative power series algebra $A:=\mathbb{Q}\langle\langle x, y\rangle\rangle$ (which is the free noncommutative complete $\mathbb{Q}$-algebra with generators $x, y)$ such that [Reu1993, Var1984]

$$
\exp (x) \exp (y)=\exp (x+y+\operatorname{BCH}(x, y)) .
$$

Let us recall the first few terms of $\operatorname{BCH}(x, y)$ which are

$$
\operatorname{BCH}(x, y)=\frac{1}{2}[x, y]+\frac{1}{12}[x,[x, y]]-\frac{1}{12}[y,[x, y]]-\frac{1}{24}[x,[y,[x, y]]]+\cdots
$$


where $[x, y]:=x y-y x$ is the commutator of $x$ and $y$ in $A$. Also denote $C(x, y):=x+y+$ $\operatorname{BCH}(x, y)$. So we have

$$
C(x, y)=\log (\exp (x) \exp (y))
$$

which is a special case of the Hausdorff series [Lod1994]

$$
Z\left(x_{1}, \ldots, x_{n}\right):=\log \left(\exp \left(x_{1}\right) \cdots \exp \left(x_{n}\right)\right) .
$$

Then for any complete $\mathbb{Q}$-algebra $A$ and $u, v \in A_{1}, C(u, v) \in A_{1}$ is well-defined. So we get a map

$$
C: A_{1} \times A_{1} \rightarrow A_{1}
$$

Now let $P: A \rightarrow A$ be any linear map preserving the filtration of $A$. We define $\tilde{P}$ to be $\operatorname{id}_{A}-P$. For $a \in A_{1}$, define $\chi(a)=\lim _{n \rightarrow \infty} \chi_{(n)}(a)$ where $\chi_{(n)}(a)$ is given by the $B C H$-recursion

$$
\begin{aligned}
\chi_{(0)}(a) & :=a, \\
\chi_{(n+1)}(a) & =a-\operatorname{BCH}\left(P\left(\chi_{(n)}(a)\right),\left(\operatorname{id}_{A}-P\right)\left(\chi_{(n)}(a)\right)\right),
\end{aligned}
$$

and where the limit is taken with respect to the topology given by the filtration. Then the map $\chi: A_{1} \rightarrow A_{1}$ satisfies

$$
\chi(a)=a-\operatorname{BCH}(P(\chi(a)), \tilde{P}(\chi(a))) .
$$

This map appeared in [EGK2004, EGK2005, EG2005], where also more details can be found. The following proposition gives further properties of the map $\chi$, improving a result in [Man2001] (in the arXiv version, Paragraph II.6.).

Proposition 1. For any linear map $P: A \rightarrow A$ preserving the filtration of $A$ there exists a unique (usually non-linear) map $\chi: A_{1} \rightarrow A_{1}$ such that $\left(\chi-\mathrm{id}_{A}\right)\left(A_{i}\right) \subset A_{2 i}$ for any $i \geq 1$, and such that, with $\tilde{P}:=\operatorname{id}_{A}-P$ we have

$$
\forall a \in A_{1}, \quad a=C(P(\chi(a)), \tilde{P}(\chi(a))) .
$$

This map is bijective, and its inverse is given by

$$
\chi^{-1}(a)=C(P(a), \tilde{P}(a))=a+\operatorname{BCH}(P(a), \tilde{P}(a)) .
$$

Proof. Equation (3) can be rewritten as

$$
\chi(a)=F_{a}(\chi(a)),
$$

with $F_{a}: A_{1} \rightarrow A_{1}$ defined by

$$
F_{a}(b)=a-\operatorname{BCH}(P(b), \tilde{P}(b)) .
$$

This map $F_{a}$ is a contraction with respect to the metric associated with the filtration: indeed if $b, \varepsilon \in A$ with $\varepsilon \in A_{n}$, we have

$$
F_{a}(b+\varepsilon)-F_{a}(b)=\operatorname{BCH}(P(b), \tilde{P}(b))-\operatorname{BCH}(P(b+\varepsilon), \tilde{P}(b+\varepsilon)) .
$$

The right-hand side is a sum of iterated commutators in each of which $\varepsilon$ does appear at least once. So it belongs to $A_{n+1}$. So the sequence $F_{a}^{n}(b)$ converges in $A_{1}$ to a unique fixed point $\chi(a)$ for $F_{a}$. 
Let us remark that for any $a \in A_{i}$, then, by a straightforward induction argument, $\chi_{(n)}(a) \in$ $A_{i}$ for any $n$, so $\chi(a) \in A_{i}$ by taking the limit. Then $\chi(a)-a=\operatorname{BCH}(P(\chi(a)), \tilde{P}(\chi(a)))$ clearly belongs to $A_{2 i}$. Now consider the map $\psi: A_{1} \rightarrow A_{1}$ defined by $\psi(a)=C(P(a), \tilde{P}(a))$. It is clear from the definition of $\chi$ that $\psi \circ \chi=\mathrm{id}_{A}$. Then $\chi$ is injective and $\psi$ is surjective. The injectivity of $\psi$ will be an immediate consequence of the following lemma

Lemma 2. The map $\psi$ increases the ultrametric distance given by the filtration.

Proof. For any $x, y \in A_{1}$ the distance $d(x, y)$ is given by $\mathrm{e}^{-n}$ where $n=\sup \left\{k \in \mathbb{N}, x-y \in A_{k}\right\}$. We have then to prove that $\psi(x)-\psi(y) \notin A_{n+1}$. But

$$
\begin{aligned}
\psi(x)-\psi(y) & =x-y+\operatorname{BCH}(P(x), \tilde{P}(x))-\operatorname{BCH}(P(y), \tilde{P}(y)) \\
& =x-y+(\operatorname{BCH}(P(x), \tilde{P}(x))-\operatorname{BCH}(P(x)-P(x-y), \tilde{P}(x)-\tilde{P}(x-y))) .
\end{aligned}
$$

The rightmost term inside the large brackets clearly belongs to $A_{n+1}$. As $x-y \notin A_{n+1}$ by hypothesis, this proves the claim.

The map $\psi$ is then a bijection, so $\chi$ is also bijective, which proves Proposition 1.

Now let $\mathfrak{g}$ be a complete filtered Lie algebra. Let $A:=\mathcal{U}(\mathfrak{g})$ be the universal enveloping associative algebra of $\mathfrak{g}$. Then with the induced filtration from $\mathfrak{g}, A$ is a complete filtered associative algebra. $A$ is also a complete Lie algebra under the bracket $[x, y]:=x y-y x$ and contains $\mathfrak{g}$ as a complete filtered sub-Lie algebra. Let $P: \mathfrak{g} \rightarrow \mathfrak{g}$ be a linear map preserving the filtration in $\mathfrak{g}$. We can extend $P$ to a linear map $\hat{P}: A \rightarrow A$ that preserves the filtration in $A$. A simple way to build such an extension (by no means unique) is to choose any supplementary subspace $V$ of $\mathfrak{g}$ inside $A$ and to extend $P$ by the identity map on the complement. If $P$ is idempotent, so is $\hat{P}$. As is well-known [Reu1993, Var1984], the power series $C(x, y)$ and $\operatorname{BCH}(x, y) \in \mathbb{Q}\langle\langle x, y\rangle\rangle$ are Lie series. Therefore, the map $\chi: A_{1} \rightarrow A_{1}$ in Eq. (2) and Proposition 1 restricts to a bijective map

$$
\chi: \mathfrak{g}_{1} \rightarrow \mathfrak{g}_{1}
$$

with its inverse given by Eq. (4). Further, for $u, v \in \mathfrak{g}_{1}, C(u, v)$ is a well-defined element in $\mathfrak{g}_{1}$. We thus have

$$
C: \mathfrak{g}_{1} \times \mathfrak{g}_{1} \rightarrow \mathfrak{g}_{1}
$$

as in the associative case.

The following theorem contains the key result of our exposition. It states a general decomposition on $A$ implied by the map $\chi$.

Theorem 3. Let $A$ be a complete filtered associative algebra or Lie algebra with a linear, filtration preserving map $P: A \rightarrow A$.

(1) For any $a \in A_{1}$, we have

$$
\exp (a)=\exp (P(\chi(a))) \exp (\tilde{P}(\chi(a))) .
$$


(2) $C: A_{1} \times A_{1} \rightarrow A_{1}$ has a right inverse $D_{P}$ given by

$$
D_{P}=(P \circ \chi, \tilde{P} \circ \chi): A_{1} \rightarrow A_{1} \times A_{1} .
$$

(3) $C$ restricts to a bijection

$$
C: D_{P}\left(A_{1}\right) \rightarrow A_{1}
$$

(4) Furthermore, for any subset $B$ of $A_{1}, C$ restricts to a bijection

$$
C: D_{P}(B) \rightarrow B
$$

Proof. (1) follows since

$$
C(P(\chi(a)), \tilde{P}(\chi(a)))=a .
$$

(2) follows since

$$
C \circ D_{P}(a)=C(P(\chi(a)), \tilde{P}(\chi(a)))=a .
$$

(3) is a general property of maps:

$$
\left.\left(D_{P} \circ C\right)\right|_{D_{P}\left(A_{1}\right)}\left(a_{1}, a_{2}\right)=\left(D_{P} \circ C\right) \circ D_{P}(a)=D_{P} \circ\left(C \circ D_{P}\right)(a)=D_{P}(a) .
$$

(4) is clear as $D_{P}$ is a (two-sided) inverse for the restriction of $C$ to $D_{P}\left(A_{1}\right)$.

The particular case when the map $P$ is idempotent deserves special attention.

Theorem 4. Let $P: A \rightarrow A$ be an idempotent linear map preserving the filtration of $A$. Let $A=A_{-} \oplus A_{+}$be the corresponding vector space decomposition, with $A_{-}:=P(A)$ and $A_{+}:=\tilde{P}(A)$. Let $A_{1,-}:=P\left(A_{1}\right)$ and $A_{1,+}:=\tilde{P}\left(A_{1}\right)$. Let $\chi: A_{1} \rightarrow A_{1}$ be the BCH-recursion map associated to the map $P$, and let $\tilde{\chi}: A_{1} \rightarrow A_{1}$ be the $B C H$-recursion map associated to $\tilde{P}$

(1) (Factorization Theorem) $C$ restricts to a bijection

$$
C_{-}: A_{1,-} \times A_{1,+} \longrightarrow A_{1} \text {. }
$$

(2) (Formal Uniformization Theorem) There exists a unique bijection

$$
\Psi: A_{1,+} \times A_{1,-} \longrightarrow A_{1,-} \times A_{1,+}
$$

such that for $a=\left(a_{+}, a_{-}\right) \in A_{1,+} \times A_{1,-}$, we have

$$
\exp \left(a_{+}\right) \exp \left(a_{-}\right)=\exp \left(\pi_{-}(\Psi(a))\right) \exp \left(\pi_{+}(\Psi(a))\right),
$$

where $\pi_{ \pm}: A_{1,-} \times A_{1,+} \rightarrow A_{1, \pm}$ are the projectors.

(3) The inverse map of $C_{-}$in part (1) is given by

$$
D_{P}(a)=(P(\chi(a)), \tilde{P}(\chi(a))), \quad a \in A_{1},
$$

and the uniformization map $\Psi$ in part (2) writes

$$
\Psi(a)=(P(\chi \circ C(a)), \tilde{P}(\chi \circ C(a)))
$$

or

$$
\Psi(a)=\left(P\left(\chi \circ \tilde{\chi}^{-1}\left(a_{+}+a_{-}\right)\right), \tilde{P}\left(\chi \circ \tilde{\chi}^{-1}\left(a_{+}+a_{-}\right)\right)\right)
$$

with $a=\left(a_{+}, a_{-}\right) \in A_{1,+} \times A_{1,-}$. 
The statements (1) and (2) in the above theorem generalize theorems of Barron, Huang and Lepowsky [BHL2000] which are themselves generalizations of factorization and uniformization theorems for Lie algebras and Lie superalgebras such as Virasoro algebras and Neveu-Schwarz algebras, respectively. This is the motivation for the naming of those items. See Section 4 for further details.

Proof. (1) We already know from item (3) of Theorem 3 that $D_{P}$ is a right inverse for $C_{-}$. But it is also a left inverse, as for any $(x, y) \in A_{1,-} \times A_{1,+}$ there is a unique $v \in A_{1}$ such that $x=P(v)$ and $y=\tilde{P}(v)$, and we have

$$
\begin{aligned}
D_{P} \circ C(x, y) & =D_{P} \circ C(P(v), \tilde{P}(v)) \\
& =D_{P} \circ \chi^{-1}(v) \\
& =(P, \tilde{P})(v) \\
& =(x, y) .
\end{aligned}
$$

(2) By the same argument as for part (1), $C$ restricts to a bijection

$$
C_{+}: A_{1,+} \times A_{1,-} \longrightarrow A_{1} \text {. }
$$

Its inverse is now $D_{\tilde{P}}$. Since $A_{1}=A_{1,+} \oplus A_{1,-}=A_{1,-} \oplus A_{1,+}$, we can define $\Psi$ by the following diagram

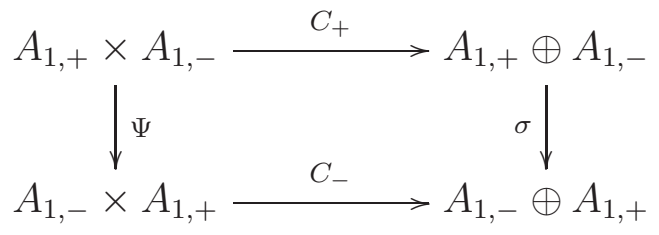

Here $\sigma$ is just a cosmetical way to write the identity map

$$
\sigma\left(b^{+}+b^{-}\right)=b^{-}+b^{+} .
$$

$\Psi$ is bijective since $C_{+}$and $C_{-}$in the diagram are. We see also from the diagram that this $\Psi$ is the unique map such that

$$
\exp \left(a_{+}\right) \exp \left(a_{-}\right)=\exp \left(\pi_{-}(\Psi(a))\right) \exp \left(\pi_{+}(\Psi(a))\right)
$$

(3) This follows from the above commutative diagram and part (1): now we can compute

$$
\begin{aligned}
\Psi(a) & =D_{P} \circ C_{+}(a) \\
& =\left(P \circ \chi \circ C_{+}(a), P \circ \chi \circ C_{+}(a)\right) \\
& =\left(P\left(\chi \circ \tilde{\chi}^{-1}\left(a_{+}+a_{-}\right)\right), \tilde{P}\left(\chi \circ \tilde{\chi}^{-1}\left(a_{+}+a_{-}\right)\right)\right)
\end{aligned}
$$

which ends the proof of Theorem 4.

Corollary 5. Under the hypotheses of Theorem 4, for any $\eta \in 1+A_{1}$ there are unique $\eta_{-} \in \exp \left(A_{1,-}\right)$ and $\eta_{+} \in \exp \left(A_{1,+}\right)$ such that $\eta=\eta_{-} \eta_{+}$.

Proof. This follows directly from the first item of Theorem 3 and the first item of Theorem 4, as the exponential map is a bijection from $A_{1}$ onto $1+A_{1}$. 
Let us finish this section with two observations simplifying the $B C H$-recursion considerably. The first one is of more general character. To begin with it might be helpful to work out the first few terms of the recursion for the map $\chi$ in (1). For this let us introduce a dummy parameter $t$ and write $\chi(a t)=t \sum_{k \geq 0} \chi^{(k)}(a) t^{k}$. For $k=0,1,2$ we readily find $\chi^{(0)}(a)=a$ and

$$
\begin{aligned}
\chi^{(1)}(a)=- & -\frac{1}{2}[P(a), \tilde{P}(a)]=-\frac{1}{2}[P(a), a] \\
\chi^{(2)}(a)=-\frac{1}{2}\left[P\left(\chi^{(1)}(a)\right), \tilde{P}(a)\right]-\frac{1}{2}\left[P(a), \tilde{P}\left(\chi^{(1)}(a)\right)\right] & -\frac{1}{12}([P(a),[P(a), a]]-[\tilde{P}(a),[P(a), a]]) \\
=+\frac{1}{4}[P([P(a), a]), \tilde{P}(a)]+ & \frac{1}{4}[P(a), \tilde{P}([P(a), a])] \\
& -\frac{1}{12}([P(a),[P(a), a]]-[\tilde{P}(a),[P(a), a]]) \\
= & \frac{1}{4}[P([P(a), a]), a]+\frac{1}{12}([P(a),[P(a), a]]-[[P(a), a], a]) .
\end{aligned}
$$

In both the last cases $\tilde{P}=\operatorname{id}_{A}-P$ has completely disappeared. Therefore, we might expect to find a simpler recursion for the map $\chi$, without the appearance of $\tilde{P}$. Indeed, such a simplification follows using the factorization property, implied by the $\chi$ map on $A$ in item (1) of Theorem 3.

Lemma 6. [EGK2005] Let $A$ be a complete filtered algebra and $P: A \rightarrow A$ a linear map preserving the filtration. The map $\chi$ in (2) solves the following recursion

$$
\chi(u):=u+\operatorname{BCH}(-P(\chi(u)), u), \quad u \in A_{1} .
$$

Proof. For any element $u \in A$ we can write $u=P(u)+\left(\operatorname{id}_{A}-P\right)(u)$ using linearity of $P$. The definition of the map $\chi$ then implies for $u \in A_{1}$ that $\exp (u)=\exp (P(\chi(u))) \exp (\tilde{P}(\chi(u)))$, see Eq. (5). Furthermore,

$$
\begin{aligned}
\exp (\tilde{P}(\chi(u))) & =\exp (-P(\chi(u))) \exp (u) \\
& =\exp (-P(\chi(u))+u+\operatorname{BCH}(-P(\chi(u)), u)) .
\end{aligned}
$$

Bijectivity of the exp map then implies that

$$
\chi(u)-P(\chi(u))=-P(\chi(u))+u+\operatorname{BCH}(-P(\chi(u)), u) .
$$

From which Equation (8) follows.

Our second observation is of more particular type. Again, it concerns the linear map $P$ in the definition of the $B C H$-recursion $\chi$. We will treat a special case, providing a solution, i.e., closed form, for the $B C H$-recursion. Further below in Section 5 we will observe another instance where a closed form for the $B C H$-recursion can be derived, see Eq. (50).

Let us now assume that the linear map $P: A \rightarrow A$ in the $B C H$-recursion in Eq. (8) of Lemma 6 is an idempotent map, and moreover that it respects multiplication in $A$. This 
makes $P$ respectively $\tilde{P}=\mathrm{id}_{A}-P$ a Rota-Baxter map to be introduced in the following section although the map $\tilde{P}$ is not an algebra morphism. We then have

Lemma 7. Let $A$ be a complete filtered associative algebra with filtration preserving linear map $P: A \rightarrow A$, which moreover is an idempotent algebra homomorphism. Then the map $\chi$ in Eq. (8) of Lemma 6 has the simple form

$$
\chi(u)=u+\operatorname{BCH}(-P(u), u),
$$

for any element $u \in A_{1}$.

Proof. The proof follows from Lemma 6, since $P(\chi(u))=P(u)$. The latter results from the multiplicativity of $P$, i.e., applying $P$ to Eq. (2) we obtain

$$
\left.P(\chi(u))=P(u)+\operatorname{BCH}\left(P^{2}(\chi(u)),(P \circ \tilde{P})(u)\right)\right) .
$$

Since $P$ is idempotent, we have $P \circ \tilde{P}=P-P^{2}=0$. Thus $P(\chi(u))=P(u)$.

Remark: With the foregoing assumptions on $P$ the factorization in item (1) of Theorem 3 simplifies considerably. For any $a \in A_{1}$, we have

$$
\exp (a)=\exp (P(a)) \exp (\tilde{P}(a)+\mathrm{BCH}(-P(a), a))
$$

2.2. Rota-Baxter operator. In the 1950 s and early $1960 \mathrm{~s}$, several interesting results were obtained in the fluctuation theory of probability. One of the most well-known is Spitzer's identity [Spit1956]. In a seminal 1960 paper [Bax1960], the American mathematician G. Baxter deduced it from a certain operator identity, that later bore his name. During the early 1960s and 1970s, algebraic, combinatorial and analytic aspects of Baxter's work were studied by several people, among them G.-C. Rota and F. V. Atkinson. Much of the recent renewed interest into these works owes to Rota's later survey articles [Rota1995, Rota1998] and talks during the 1990s. Related concepts were independently developed by Russian physicists during the 1980s. Especially in Belavin and Drinfeld's, and Semenov-Tian-Shansky's papers [BelDri1982, Sem1983] on solutions of the (modified) classical Yang-Baxter equation. In this context let us mention another connection linked with the last remark. Aguiar [Agu2000] related Rota-Baxter operators of weight zero to the associative analog of the classical YangBaxter equation, which also appeared in [Poli2002].

Now we assume that $A$ is an associative algebra and $P$ a Rota-Baxter operator of weight $\theta$ satisfying the Rota-Baxter relation

$$
P(x) P(y)+\theta P(x y)=P(x P(y))+P(P(x) y)
$$

for all $x, y \in A$ [Bax1960, Rota1969, Rota1995, RoSm1972]. A Rota-Baxter algebra of weight $\theta$ is an algebra with a Rota-Baxter operator denoted by the pair $(A, P)$. The operator $\tilde{P}:=$ $\theta \operatorname{id}_{A}-P$ also is a Rota-Baxter map of weight $\theta$, such that the mixed relation

$$
P(x) \tilde{P}(y)=\tilde{P}(P(x) y)+P(x \tilde{P}(y))
$$


is satisfied for all $x, y \in A$. The image of $P$ as well as $\tilde{P}$ are subalgebras in $A$. A Rota-Baxter ideal $I$ is an ideal $I$ of $A$ such that $P(I) \subseteq I$.

The case $\theta=0$ corresponds to the integration by parts property of the usual Riemann integral. An important class of examples is given by idempotent Rota-Baxter maps, i.e., projectors, where identity (11) (of weight $\theta=1$ ) implies that the Rota-Baxter algebra $A$ splits as a direct sum into two parallel subalgebras given by the image, respectively kernel, of $P$. Assuming $P$ to be an idempotent algebra morphism is sufficient to imply that it is a RotaBaxter map. As an example of an idempotent Rota-Baxter map which is moreover an algebra morphism, truncate the Taylor expansion of a real function at a point $a$ at zeroth order, i.e., evaluate a real function at a point $a, P_{a}^{(0)}(f)(x)=f(a)$.

The modified Rota-Baxter operator, $B:=\theta \mathrm{id}_{A}-2 P$, satisfies the modified Rota-Baxter relation

$$
B(x) B(y)+\theta^{2} x y=B(B(x) y+x B(y)) .
$$

for all $x$ and $y$ in $A$. For a modified Rota-Baxter operator $B$ coming from an idempotent Rota-Baxter map $P$, we have $B^{2}=\operatorname{id}_{A}, B \circ P=-P$, and $B \circ \tilde{P}=\tilde{P}$.

Taking the Lie algebra associated to $(A, P)$, with commutator bracket $[x, y]:=x y-y x$, for all $x, y \in A$, we find the Rota-Baxter Lie algebra, $\left(L_{A}, P\right)$, of weight $\theta$ with $P$ fulfilling

$$
[P(x), P(y)]+\theta P([x, y])=P([P(x), y]+[x, P(y)]) .
$$

Similarly, for the modified Rota-Baxter map. Both equations are known as (the operator form of) the (modified) classical Yang-Baxter ${ }^{1}$ equations [BelDri1982, Sem1983].

Every Rota-Baxter algebra $(A, P)$ of weight $\theta$ allows for a new product defined in terms of the Rota-Baxter map $P$

$$
a *_{P} b:=P(a) b+a P(b)-\theta a b
$$

such that the vector space $A$ with this product is a Rota-Baxter algebra of the same weight, with $P$ as its Rota-Baxter map. We will denote it by $\left(A_{P}, P\right)$. The Rota-Baxter map $P$ becomes an (not necessarily unital) algebra homomorphism from $A_{P}$ to $A, P\left(a *_{P} b\right)=P(a) P(b)$. For $\tilde{P}$ we have $\tilde{P}\left(a *_{P} b\right)=-\tilde{P}(a) \tilde{P}(b)$.

A complete filtered Rota-Baxter algebra is defined to be a Rota-Baxter algebra $(A, P)$ with a complete filtration by Rota-Baxter ideals $\left\{A_{n}\right\}$. Again, consider for any weight $\theta$ RotaBaxter algebra $(A, P)$ the power series $\operatorname{ring} \mathcal{A}:=A[[t]]$ and define an operator $\mathcal{P}: \mathcal{A} \rightarrow \mathcal{A}$, $\mathcal{P}\left(\sum_{n=0}^{\infty} a_{n} t^{n}\right):=\sum_{n=0}^{\infty} P\left(a_{n}\right) t^{n}$. Then $(\mathcal{A}, \mathcal{P})$ is a complete filtered Rota-Baxter algebra of weight $\theta$. In the case of the algebra of strictly (upper) lower triangular matrices $\mathcal{M}_{n}^{\ell}(A)$ with $n \leq \infty$ and entries in a weight $\theta$ Rota-Baxter algebra $(A, P)$, define the Rota-Baxter map $\mathcal{P}: \mathcal{M}_{n}^{\ell}(A) \rightarrow \mathcal{M}_{n}^{\ell}(A)$ entrywise, $\mathcal{P}(\alpha)=\left(P\left(\alpha_{i j}\right)\right)$, for $\alpha$ in $\mathcal{M}_{n}^{\ell}(A)$ [EG2005].

The normalized map $\theta^{-1} P$ is a Rota-Baxter operator of weight one. In the following we will assume that any Rota-Baxter map is of weight one, if not stated otherwise. The next

\footnotetext{
${ }^{1}$ Refereing to the Australian physicist Rodney Baxter.
} 
proposition contains the generalization of Spitzer's identity to non-commutative Rota-Baxter algebras.

Proposition 8. [EGK2004, EGK2005, EG2005] Let $(A, P)$ be a complete filtered Rota-Baxter algebra. The factors on the right hand side of Equation (5)

$$
\exp (a)=\exp (P(\chi(a))) \exp (\tilde{P}(\chi(a)))
$$

for $a \in A_{1}$ are the unique solutions to the equations

$$
u=1-P(\check{b} u) \quad \text { resp. } u^{\prime}=1-\tilde{P}\left(u^{\prime} \check{b}\right),
$$

where $\breve{b}:=\exp (-a)-1$ in $A_{1}$. Its inverses satisfy uniquely the equations

$$
x=1-P\left(\begin{array}{ll}
x & b
\end{array}\right) \quad \text { resp. } x^{\prime}=1-\tilde{P}\left(b x^{\prime}\right),
$$

where $b:=\exp (a)-1=(1+\check{b})^{-1}-1 \in A_{1}$.

The following theorem is due to Atkinson [Atk1963].

Theorem 9. For the solutions $x$ and $x^{\prime}$ in (18) (resp. its inverses in (17)) with $b:=\exp (a)-1$ we have

$$
x(1+b) x^{\prime}=1 \text {, that is, }(1+b)=x^{-1} x^{\prime-1} .
$$

If $P$ is idempotent, i.e., the algebra $A$ decomposes directly into the images of $P$ and $\tilde{P}$, the factorization of $1+b$ is unique.

The next corollary follows readily and is stated for completeness.

Corollary 10. Let $(A, P)$ be a complete filtered Rota-Baxter algebra. For the solutions $u$ and $u^{\prime}$ in (17), we find the equations

$$
u=1+P\left(b x^{\prime}\right), \quad u^{\prime}=1+\tilde{P}(x b),
$$

where $x$ and $x^{\prime}$ are solutions of Eqs. (18), respectively.

As a proposition we mention without giving further details the fact that, using the double Rota-Baxter product $*_{P}$ in (15) for $\theta=1$, we may write

$$
x=1+P\left(\exp ^{* P}(-\chi(a))-1\right),
$$

where $\exp ^{* P}$ denotes the exponential defined in terms of the product in (15). This implies $-x b=\exp ^{* P}(-\chi(\log (1+b)))-1$ for $1+b:=\exp (a)$.

When $(A, P)$ is commutative, the map $\chi$ reduces to the identity map, giving back Spitzer's classical identity, for fixed $b \in A_{1}$ [Spit1956]

$$
\exp (-P(\log (1+b)))=\sum_{n=0}^{\infty}(-1)^{n} \underbrace{P(P(\cdots P(P}_{n-\text { times }}(b) b) \ldots b) b)
$$

corresponding to the first recursion, $x=1-P(x b)$, in (18). Replacing the Rota-Baxter map $P$ by the identity map, the above identity reduces to the geometric series for the element $-b \in A_{1}$. Proofs of this identity in the commutative case have been given by quite a few 
authors, including the aforementioned Atkinson [Atk1963], Cartier [Car1972], Kingman and Wendel [King1962, Wen1962] as well as Rota and Smith [RoSm1972]. In fact, Rota [Rota1969] showed that this identity is equivalent to the classical Waring identity relating elementary symmetric functions and power symmetric functions.

Remark: Coming back to Lemma 7, respectively Eq. (10) we see immediately that in the case of a non-commutative Rota-Baxter algebra $(A, P)$ with idempotent and multiplicative RotaBaxter map $P$ and thence necessarily of weight one, implying $P(\chi(a))=P(a)$, for all $a \in A_{1}$, we have the surprising result that the exponential solution to the recursion $x=1-P(x b)$ in (18) can be written as a geometric series

$$
\exp (-P(\chi(\log (1+b))))=\exp (-P(\log (1+b)))=\frac{1}{1+P(b)} .
$$

Observe that the $B C H$-recursion $\chi$ disappeared after the first equality, since $P(\chi(a))=P(a)$.

The normalization of the weight one Rota-Baxter map $P$ to $\theta P$ gives a Rota-Baxter map of weight $\theta$. This implies the following modification of Proposition 8.

Proposition 11. For a weight $\theta \neq 0$ Rota-Baxter operator $P$, the map $\chi$ in factorization (16) of Proposition 8 generalizes to

$$
\chi_{\theta}(a)=a-\frac{1}{\theta} \operatorname{BCH}\left(P\left(\chi_{\theta}(a)\right), \tilde{P}\left(\chi_{\theta}(a)\right)\right) .
$$

Similarly the recursion in Eq. (8) of Lemma 6 transposes into

$$
\chi_{\theta}(a)=a+\frac{1}{\theta} \mathrm{BCH}\left(-P\left(\chi_{\theta}(a)\right), \theta a\right), \quad a \in A_{1} .
$$

Such that for all $a \in A_{1}$ we have the decomposition

$$
\exp (\theta a)=\exp \left(P\left(\chi_{\theta}(a)\right)\right) \exp \left(\tilde{P}\left(\chi_{\theta}(a)\right)\right) .
$$

The factors on the right hand side of Equation (25) are inverses of the unique solutions of the equations

$$
x=1-P(x b) \quad \text { resp. } \quad x^{\prime}=1-\tilde{P}\left(b x^{\prime}\right),
$$

where $1+\theta b:=\exp (\theta a)$ in $A$.

From this proposition we arrive at

Corollary 12. Spitzer's identity for a complete filtered non-commutative Rota-Baxter algebra $(A, P)$ of weight $\theta \neq 0$ is

$$
\exp \left(-P\left(\chi_{\theta}\left(\frac{\log (1+\theta b)}{\theta}\right)\right)\right)=\sum_{n=0}^{\infty}(-1)^{n} \underbrace{P(b P(b P(b \cdots P}_{n-\text { times }}(b)) \ldots)),
$$

for $b \in A_{1}$. 
We call $\chi_{\theta}$ the $B C H$-recursion of weight $\theta \in \mathbb{K}$, or simply $\theta$ - $B C H$-recursion. As we will see in the next part, the particular appearance of the weight $\theta$ in Eqs. $(23,24)$ reflects the fact that in the case of weight $\theta=0$, hence $\tilde{P}=-P$, Atkinson's factorization formula (19) in Theorem 9 collapses to

$$
x x^{\prime}=(1-P(x b))\left(1+P\left(b x^{\prime}\right)\right)=1
$$

for any $b \in A_{1}$, which is in accordance with (26) for $\theta \rightarrow 0$.

Remark: It should be clear that the decomposition in Eq. (25) in the above proposition is true for any complete filtered algebra $A$ with filtration preserving linear map $P$ and $\tilde{P}_{\theta}:=\theta \operatorname{id}_{A}-P$. Hence, Eqs. (23-24) generalize Theorem 3. The Rota-Baxter property only enters in the last part with respect to the equations in (26), respectively Corollary 12.

2.3. The case of vanishing weight and the Magnus recursion. Regarding Eq. (28) in connection with the factorization in Eq. (25) for a weight $\theta \neq 0$ Rota-Baxter algebra, it is interesting to observe the limit of $\theta$ going to zero in formula (24) for the $\theta$ - $B C H$-recursion. The terms in the BCH series on the right hand side of (24) vanish except for those which are linear with respect to the second variable. In general we may write $C(a, b)=a+b+\mathrm{BCH}(a, b)$ as a sum [Reu1993]

$$
C(a, b)=\sum_{n \geq 0} H_{n}(a, b)
$$

where $H_{n}(a, b)$ is the part of $C(a, b)$ which is homogenous of degree $n$ with respect to $b$. Especially, $H_{0}(a, b)=a$. For $n=1$ we have

$$
H_{1}(a, b)=\frac{\operatorname{ad} a}{1-\mathrm{e}^{-\operatorname{ad} a}}(b) .
$$

(see e.g. [Go1982]). Hence we get a non-linear map $\chi_{0}$ inductively defined on the pro-nilpotent Lie algebra $A_{1}$ by the formula

$$
\begin{aligned}
\chi_{0}(a) & =-\frac{\operatorname{ad} P\left(\chi_{0}(a)\right)}{1-\mathrm{e}^{\operatorname{ad} P\left(\chi_{0}(a)\right)}}(a) \\
& =\left(1+\sum_{n>0} b_{n}\left[\operatorname{ad} P\left(\chi_{0}(a)\right)\right]^{n}\right)(a)
\end{aligned}
$$

where $P$ is now a weight zero Rota-Baxter operator. We call this the weight zero $B C H$ recursion. The coefficients $b_{n}:=\frac{B_{n}}{n !}$ where $B_{n}$ are the Bernoulli numbers. For $n=1,2,3,4$ we find the numbers $b_{1}=-1 / 2, b_{2}=1 / 12, b_{3}=0$ and $b_{4}=-1 / 720$. The first three terms in (30) are

$$
\chi_{0}(a)=a-\frac{1}{2}[P(a), a]+\left(\frac{1}{4}[P([P(a), a]), a]+\frac{1}{12}[P(a),[P(a), a]]\right)+\cdots
$$

As a particular example we assume $P$ to be the Riemann integral operator defined by $P\{a\}(x):=\int_{0}^{x} a(y) d y$, which is a Rota-Baxter map of weight zero, i.e., it satisfies the integration by parts rule

$$
P\left\{a_{1}\right\}(x) P\left\{a_{2}\right\}(x)=P\left\{a_{1} P\left\{a_{2}\right\}\right\}(x)+P\left\{P\left\{a_{1}\right\} a_{2}\right\}(x) .
$$


The functions $a_{i}=a_{i}(x), i=1,2$ are defined over $\mathbb{R}$ and supposed to take values in a noncommutative algebra, say, matrices of size $n \times n$. Then we find

$P\left\{\chi_{0}(a)\right\}(x)=P\{a\}(x)-\frac{1}{2} P\{[P\{a\}, a]\}(x)+\frac{1}{4} P\{[P\{[P\{a\}, a]\}, a]\}(x)$

$$
+\frac{1}{12} P\{[P\{a\},[P\{a\}, a]]\}(x)+\cdots
$$

Let us write the terms in (32) explicitly

$$
\begin{aligned}
& P\{a\}(x)=\int_{0}^{x} a(y) d y \\
& \frac{1}{2} P\{[P\{a\}, a]\}(x)=\frac{1}{2} \int_{0}^{x} \int_{0}^{y_{1}}\left[a\left(y_{2}\right), a\left(y_{1}\right)\right] d y_{2} d y_{1} \\
& \frac{1}{4} P\{[P\{[P\{a\}, a]\}, a]\}(x)=\frac{1}{4} \int_{0}^{x} \int_{0}^{y_{1}} \int_{0}^{y_{2}}\left[\left[a\left(y_{3}\right), a\left(y_{2}\right)\right], a\left(y_{1}\right)\right] d y_{3} d y_{2} d y_{1} \\
& \frac{1}{12} P\{[P\{a\},[P\{a\}, a]]\}(x)=\frac{1}{12} \int_{0}^{x} \int_{0}^{y_{1}} \int_{0}^{y_{1}}\left[a\left(y_{3}\right),\left[a\left(y_{2}\right), a\left(y_{1}\right)\right]\right] d y_{3} d y_{2} d y_{1} .
\end{aligned}
$$

Baxter's original motivation was to generalize the integral equation

$$
f(x)=1+P\{f a\}(x)
$$

corresponding to the first order initial value problem

$$
\frac{d}{d x} f(x)=a(x) f(x), \quad f(0)=1
$$

with unique solution

$$
f(x)=\exp (P\{a\}(x))
$$

by replacing the Riemann integral by another Rota-Baxter map $P$ of non-zero weight $\theta$ (11) on a commutative algebra. The result is the classical Spitzer identity (21), which in the more general non-zero weight $\theta$ case takes the form

$$
\exp \left(-P\left(\frac{\log (1-\theta a)}{\theta}\right)\right)=\sum_{n=0}^{\infty} \underbrace{P(P(P(\cdots P}_{n \text {-times }}(a) a) \ldots a) a) .
$$

This follows form (27) with $b=-a$, since $\chi_{\theta}=\mathrm{id}_{A}$ in the commutative case. One readily verifies that the left-hand side of this identity reduces to the exponential exp $(P(a))$, compare with (39), in the limit $\theta \rightarrow 0$.

To summarize, Proposition 11 generalizes Proposition 8 to non-commutative weight $\theta \neq 0$ Rota-Baxter algebras. Corollary 12 describes an extension of Baxter's result on Spitzer's identity to general associative Rota-Baxter algebras of weight $\theta \neq 0$, i.e., not necessarily commutative. The particular case of vanishing weight $\theta \rightarrow 0$ is captured by the following

Lemma 13. Let $(A, P)$ be a complete filtered Rota-Baxter algebra of weight zero. For a $\in A_{1}$ the weight zero $B C H$-recursion $\chi_{0}: A_{1} \rightarrow A_{1}$ is given by the recursion in Eq. (29)

$$
\chi_{0}(a)=-\frac{a d P\left(\chi_{0}(a)\right)}{1-\mathrm{e}^{\operatorname{ad} P\left(\chi_{0}(a)\right)}}(a)
$$


(1) The equation $x=1-P\left(x\right.$ a) has a unique solution $x=\exp \left(-P\left(\chi_{0}(a)\right)\right)$.

(2) The equation $y=1+P($ a $y)$ has a unique solution $y=\exp \left(P\left(\chi_{0}(a)\right)\right)$.

Atkinson's factorization for the weight zero case, Eq. (28), follows immediately from the preceding lemma.

In view of example (32) the last lemma leads to the following corollary. Recall the work by Magnus [Mag1954] on initial value problems of the above type but in a non-commutative setting, e.g., for matrix-valued functions. He proposed an exponential solution

$$
F(x)=\exp (\Omega[a](x))
$$

with $\Omega[a](0)=0$, for the first order initial value problem $\frac{d}{d x} F(x)=a(x) F(x), F(0)=1$, respectively the corresponding integral equation $F(x)=1+P\{a F\}(x)$, where $P$ is again, of course, the Riemann integral operator. He found an expansion for $\Omega[a](x)=\sum_{n>0} \Omega^{(n)}[a](x)$ in terms of multiple integrals of nested commutators, and provided a recursive equation for the terms $\Omega^{(n)}[a](x)$ :

$$
\frac{d}{d x} \Omega[a](x)=\frac{\operatorname{ad} \Omega[a]}{\mathrm{e}^{\operatorname{ad} \Omega[a]}-1}(a)(x) .
$$

Comparison with (29) (and also (30) and (31)) settles the link between Magnus recursion and $\mathrm{BCH}$-recursion in the context of a vanishing Rota-Baxter weight, namely

Corollary 14. Let $A$ be a function algebra over $\mathbb{R}$ with values in an operator algebra. $P$ denotes the indefinite Riemann integral operator. Magnus' $\Omega$ expansion is given by the formula

$$
\Omega[a](x)=P\left(\chi_{0}(a)\right)(x) .
$$

Hence, the $\theta$ - $B C H$-recursion (23) generalizes Magnus' expansion to general weight $\theta \neq 0$ Rota-Baxter operators $P$ by replacing the weight zero Riemann integral in $F=1+P\{a F\}$.

The following commutative diagram (45) summarizes the foregoing relations. Generalizing the simple initial value problem in (38) twofold. First we go to the integral equation in (37). Then we replace the Riemann integral by a general Rota-Baxter map and assume a noncommutative setting.

Hence, we start with a complete filtered non-commutative associative Rota-Baxter algebra $(A, P)$ of non-zero weight $\theta \in \mathbb{K}$. The top of (45) contains the solution to the recursive equation

$$
y=1+P(y b)
$$

for $b \in A_{1}$ which is given in terms of Spitzer's identity generalized to associative otherwise arbitrary Rota-Baxter algebras (27),

$$
y=\exp \left(-P\left(\chi_{\theta}\left(\frac{\log (1-\theta b)}{\theta}\right)\right)\right) .
$$

The $\theta$ - $B C H$-recursion $\chi_{\theta}$ is given in (24). The left wing of (45) describes the case when first, the weight $\theta$ goes to zero, hence reducing $\chi_{\theta} \rightarrow \chi_{0}$. This is the algebraic structure underlying Magnus' $\Omega$-expansion. Then the algebra $A$ becomes commutative which implies $\chi_{0}=\mathrm{id}_{A}$. The right wing of diagram (45) just describes the opposite reduction, i.e., we fist make the algebra 
commutative, which gives the classical Spitzer identity for non-zero weight commutative RotaBaxter algebras (40). Then we take the limit $\theta \rightarrow 0$.

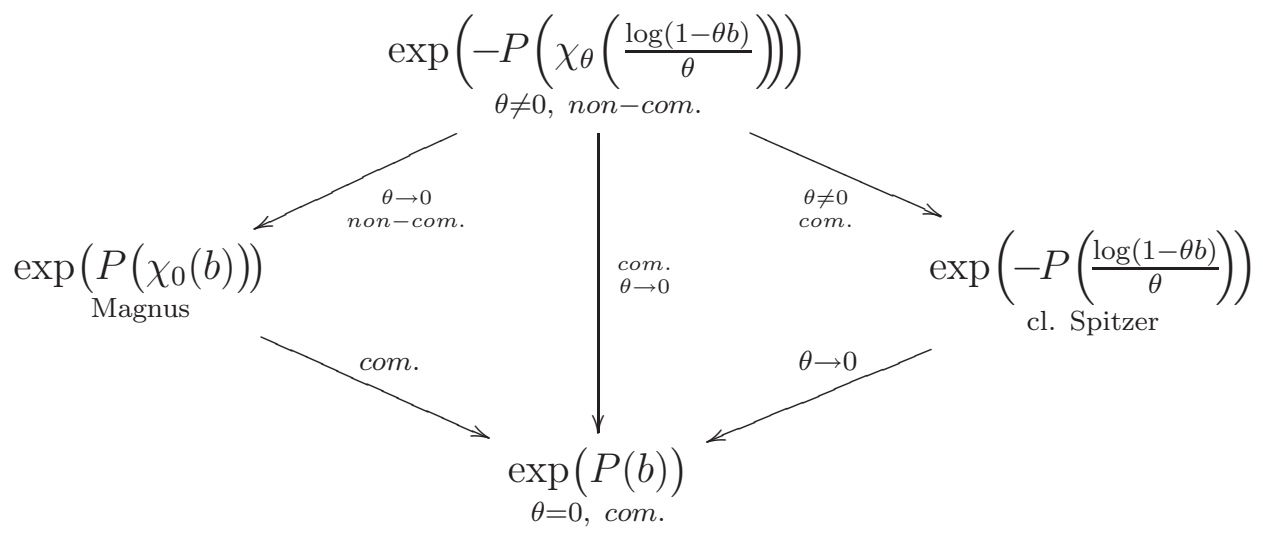

Both paths eventually arrive at the simple fact that equation (43) is solved by a simple exponential in a commutative weight zero Rota-Baxter setting. This is the general algebraic structure underlying the the initial value problem in (38) respectively its corresponding integral equation (37).

\section{Renormalization in PeRturbative QFT}

This section recalls some of the results from [EGK2004, EGK2005]. We derive Connes' and Kreimer's Birkhoff decomposition of Hopf algebra characters with values in a commutative unital Rota-Baxter algebra. For more details we refer the reader to [EG2005, EK2005, FGB2005, Man2001].

In most of the interesting and relevant 4-dimensional quantum field theories (QFT), to perform even simple perturbative calculations, one can not avoid facing ill-defined integrals. The removal of these (ultraviolet) divergencies, or short-distance singularities, in a physically and mathematically sound way is the process of renormalization [Col1984].

In the theory of Kreimer [Krei1998], and Connes and Kreimer Feynman graphs as the main building blocks of perturbative QFT are organized into a Hopf algebra. In particular, Connes and Kreimer discovered a Birkhoff type decomposition for Hopf algebra characters with values in the field of Laurent series, which captures the process of renormalization. We will briefly outline an algebraic framework for this decomposition based on the above results of Spitzer and Atkinson.

We work in the setting of Connes and Kreimer [CK2000]. Recall that in the language of Kreimer for a given perturbative renormalizable QFT, denoted by $\mathcal{F}$, we have a graded, connected, commutative, non-cocommutative Hopf algebra $\mathcal{H}_{\mathcal{F}}:=\left(\mathcal{H}:=\bigoplus_{n \geq 0} \mathcal{H}_{n}, \Delta, m_{\mathcal{H}}, \varepsilon_{\mathcal{H}}, S\right)$ of one-particle irreducible (1PI) Feynman graphs with coproduct $\Delta$ defined by

$$
\Delta(\Gamma)=\Gamma \otimes 1+1 \otimes \Gamma+\sum_{\gamma \subset \Gamma} \gamma \otimes \Gamma / \gamma
$$

Here the sum is over all 1PI ultraviolet divergent subgraphs $\gamma$ in $\Gamma$ and $\Gamma / \gamma$ denotes the corresponding cograph. The decomposition of $\Gamma$ in $\Delta(\Gamma)$ essentially describes the combinatorics of renormalization. 
The space $\operatorname{Hom}\left(\mathcal{H}_{\mathcal{F}}, \mathbb{C}\right)$ of linear maps $\mathcal{H}_{\mathcal{F}} \rightarrow \mathbb{C}$ equipped with the convolution product $f \star$ $g:=m_{\mathcal{H}} \circ(f \otimes g) \circ \Delta$ is an associative algebra with the counit $\varepsilon_{\mathcal{H}}$ as unit. $\operatorname{Hom}\left(\mathcal{H}_{\mathcal{F}}, \mathbb{C}\right)$ contains the group $G:=\operatorname{Char}\left(\mathcal{H}_{\mathcal{F}}, \mathbb{C}\right)$ of Hopf algebra characters, i.e., algebra homomorphisms, and its corresponding Lie algebra $\mathfrak{g}:=\mathfrak{g}_{\mathcal{F}}=\partial \operatorname{Char}\left(\mathcal{H}_{\mathcal{F}}, \mathbb{C}\right)$ of derivations (infinitesimal characters). Feynman rules in $\mathcal{F}$ provide such an algebra homomorphism from $\mathcal{H}_{\mathcal{F}}$ to $\mathbb{C}$.

In general, ultraviolet divergencies demand a regularization prescription, where by introducing extra parameters, the characters become algebra homomorphisms, say for instance, into $\left.L=\mathbb{C}\left[\epsilon^{-1}, \epsilon\right]\right]$, the field of Laurent series (dimensional regularization scheme). We denote by $G_{L}:=\operatorname{Char}\left(\mathcal{H}_{\mathcal{F}}, L\right) \subset \operatorname{Hom}\left(\mathcal{H}_{\mathcal{F}}, L\right)$ the group of $L$-valued, or regularized, algebra homomorphisms. Hence, now the set of Feynman rules together with dimensional regularization amounts to a linear map from the set of 1PI Feynman graphs to $L$ and hence an algebra homomorphism denoted by $\phi \in G_{L}$ from $\mathcal{H}_{\mathcal{F}}$ to $L$.

We will now make the connection to subsection 2.2. The field of Laurent series actually forms a commutative Rota-Baxter algebra $(L, R)$ with the projector $R$ on $L$

$$
R: L \rightarrow L, \sum_{i=-n}^{\infty} a_{i} \epsilon^{i} \mapsto \sum_{i=-n}^{-1} a_{i} \epsilon^{i}
$$

to the strict pole part of a Laurent series as the idempotent weight-one Rota-Baxter map (minimal subtraction scheme).

It was shown in [CK2000, Krei1998] that this setup allows for a concise Hopf algebraic description of the process of perturbative renormalization of the QFT $\mathcal{F}$. To wit, Connes and Kreimer observed that Bogoliubov's recursive formula for the counter term in renormalization has a Hopf algebraic expression given by inductively defining the map $\phi_{-} \in \mathcal{H}_{\mathcal{F}}$

$$
\phi_{-}(\Gamma)=-R\left(\phi(\Gamma)+\sum_{\gamma \subset \Gamma} \phi_{-}(\gamma) \phi(\Gamma / \gamma)\right)
$$

with $\phi_{-}(\Gamma)=-R(\Gamma)$ if $\Gamma$ is a primitive element in $\mathcal{H}_{\mathcal{F}}$, i.e., contains no subdivergence. The map

$$
\overline{\mathrm{R}}[\phi](\Gamma):=\phi(\Gamma)+\sum_{\gamma \subset \Gamma} \phi_{-}(\gamma) \phi(\Gamma / \gamma)
$$

for $\Gamma \in \operatorname{ker}\left(\varepsilon_{\mathcal{H}}\right)$ is Bogoliubov's preparation map. This lead to the Birkhoff decomposition of Feynman rules found by Connes and Kreimer [CK1999, CK2000, CK2001, Krei1999], described in the following theorem.

Theorem 15. The renormalization of $\phi \stackrel{\text { ren. }}{\longrightarrow} \phi_{+}$follows from the convolution product of the counter term $\phi_{-}$(46) with $\phi, \phi_{+}:=\phi_{-} \star \phi$, implying the inductive formula for $\phi_{+}$

$$
\phi_{+}(\Gamma)=\phi(\Gamma)+\phi_{-}(\Gamma)+\sum_{\gamma \subset \Gamma} \phi_{-}(\gamma) \phi(\Gamma / \gamma)
$$

Further, the maps $\phi_{-}$and $\phi_{+}$are the unique characters such that $\phi=\phi_{-}^{-1} \star \phi_{+}$gives the algebraic Birkhoff decomposition of the regularized Feynman rules character $\phi \in G_{L}$.

The following theorem describes the Birkhoff decomposition of Connes and Kreimer in Theorem 15 using the algebraic setting developed in the earlier sections. 
Theorem 16. [EGK2004, EGK2005] In Proposition 8, take $A$ to be $\left(\operatorname{Hom}\left(\mathcal{H}_{\mathcal{F}}, L\right), \mathcal{R}\right)$, which is a complete filtered Rota-Baxter algebra with Rota-Baxter operator $\mathcal{R}(\phi):=R \circ \phi$ and filtration from $\mathcal{H}$. We denote its unit by $e:=u_{L} \circ \varepsilon_{\mathcal{H}}$. For a L-valued character $\phi \in \operatorname{Char}\left(\mathcal{H}_{\mathcal{F}}, L\right)$ take $b:=\phi-e$. Then one can show that $b \in A_{1}$ and

(1) the equations in (18) are the recursive formulae for $x=: \phi_{-}$and $x^{\prime}=: \phi_{+}^{-1}$ in the work of Connes-Kreimer;

(2) the exponential factors in Equation (5) give the unique explicit formulae for $x^{-1}=\phi_{-}^{-1}$ and $x^{-1}=\phi_{+}$.

(3) equation (5) gives the unique Birkhoff decomposition of $\phi=\phi_{-}^{-1} \star \phi_{+}$found in ConnesKreimer's work;

(4) Bogoliubov's $\overline{\mathrm{R}}-$ map, $\overline{\mathrm{R}}[\phi]: \mathcal{H} \rightarrow L$, is given by $\overline{\mathrm{R}}[\phi]=\exp ^{\star \mathcal{R}}(-\chi(\log (\phi)))$, for $\phi \in G_{L}$, such that $\tilde{\mathcal{R}}(\overline{\mathrm{R}}[\phi])=2 e-\phi_{+}$and $\mathcal{R}(\overline{\mathrm{R}}[\phi])=\phi_{-}-e$. Here $\phi_{1} \star_{\mathcal{R}} \phi_{2}:=\mathcal{R}\left(\phi_{1}\right) \star \phi_{2}+\phi_{1} \star$ $\mathcal{R}\left(\phi_{2}\right)-\phi_{1} \star \phi_{2}, \phi_{i} \in G_{L}, i=1,2$, see (15).

It is evident that one can replace the particular choice of the field of Laurent series $L$ by any other commutative Rota-Baxter algebra with idempotent Rota-Baxter map. Proposition

8 provides us with a recursion for the renormalization of $\phi \stackrel{\text { ren. }}{\longrightarrow} \phi_{+}$which does not contain the counter term $\phi_{-}$.

Corollary 17. [EGK2004, EG2005, EGGV2006] With the assumption of Theorem 16, the second equation in (17) gives a recursion for $\phi_{+}$

$$
\phi_{+}=e-\tilde{\mathcal{R}}\left(\phi_{+} \star\left(\phi^{-1}-e\right)\right) .
$$

Recall that the inverse of $\phi \in G_{L}$ is given by the composition with the antipode, $\phi^{-1}=\phi \circ S$.

We should mention that in recent work [EGGV2006, EG2005] the first two authors showed, together with J. M. Gracia-Bondía and J. C. Várilly, how the combinatorics of perturbative renormalization can be represented by matrix factorization of unipotent upper (lower) triangular matrices with entries in a commutative Rota-Baxter algebra. As we have seen above such triangular matrices provide a simple example of a complete filtered Rota-Baxter algebra.

\section{Formal Exponentials}

In this section we consider now the factorization of formal exponentials described by Barron, Huang and Lepowsky in [BHL2000] in the context of the BCH-recursion map $\chi$. Let us first recall their notations and results.

Let $\mathfrak{g}$ be a Lie algebra with a decomposition $\mathfrak{g}^{-} \oplus \mathfrak{g}^{+}$of the underlying vector space. Equivalently, there is an idempotent linear map $P: \mathfrak{g} \rightarrow \mathfrak{g}$. Then we have the corresponding decomposition of the complete Lie algebra

$$
\mathfrak{g}[[s, t]]=\mathfrak{g}^{-}[[s, t]] \oplus \mathfrak{g}^{+}[[s, t]] .
$$

Consider the (restriction of the) BCH map

$$
C: \mathfrak{g}[[s, t]]_{1} \times \mathfrak{g}[[s, t]]_{1} \rightarrow \mathfrak{g}[[s, t]]_{1} .
$$


Here $\mathfrak{g}[[s, t]]_{1}=s \mathfrak{g}[[s, t]]+t \mathfrak{g}[[s, t]]$.

Theorem 18. [BHL2000]

(1) (Factorization Theorem of Barron-Huang-Lepowsky) The map

$$
C: s \mathfrak{g}^{-}[[s, t]] \times t \mathfrak{g}^{+}[[s, t]] \rightarrow s \mathfrak{g}^{-}[[s, t]] \oplus t \mathfrak{g}^{+}[[s, t]]
$$

is bijective. Here we have direct product on the source space and (direct) sum in $\mathfrak{g}[[s, t]]$ on the target space.

(2) (Formal Algebraic Uniformization of Barron-Huang-Lepowsky) There exists a unique bijection

$$
\Psi=\left(\Psi_{-}, \Psi_{+}\right): t \mathfrak{g}^{+}[[s, t]] \times s \mathfrak{g}^{-}[[s, t]] \rightarrow s \mathfrak{g}^{-}[[s, t]] \times t \mathfrak{g}^{+}[[s, t]]
$$

such that for $g^{ \pm} \in \mathfrak{g}^{ \pm}[[s, t]]$,

$$
\exp \left(t g^{+}\right) \exp \left(s g^{-}\right)=\exp \Psi_{-}\left(t g^{+}, s g^{-}\right) \exp \Psi_{+}\left(t g^{+}, s g^{-}\right) .
$$

They further posed the following problem.

Problem 19. [BHL2000, Problem 3.2] Find a closed form for the inverse map of $C$ in Theorem 18.(1).

The theorem of Barron, Huang and Lepowsky generalizes a well-known result in the case when $\mathfrak{g}$ is a finite-dimensional Lie algebra over $\mathbb{R}$ or $\mathbb{C}$. In this special case, the proof was obtained by a geometric argument on the corresponding Lie group. Actually, their proof is algebraic, making use of a more precise expression of $C(x, y)$ and the gradings given by $s$ and $t$. We will show here how to derive this result from Theorem 4.

Theorem 20. (1) The bijection $C$ in Theorem 18.(1) is the restriction $C_{-}$of the bijection $C$ in part (1) of Theorem 4.

(2) The uniformization $\Psi$ in Theorem 18.(2) is the restriction of the uniformization $\Psi$ in Theorem 4.(2).

(3) The restriction of the formulae for $C_{-}$and $\Psi$ in Theorem 4.(3) gives the formulae for the inverse map of $C$ and $\Psi$ in Theorem 18.(1).

Remark: The formulae in item (3) involve the $B C H$-recursion $\chi$ which is defined in terms of the recursive equation (2), respectively (8). Hence, our approach allows for a compact formulation of Problem 19 in a generalized setting, to wit, find a closed form for the recursively defined map $\chi$. Moreover, Lemma 7 and especially Equation (50) of Section 5 give solutions to the $\mathrm{BCH}$-recursion $\chi$, that is, closed forms for the inverse map of $C$, some particular situation.

Proof. (1) We first note that $\overline{\mathfrak{g}}:=\mathfrak{g}[[s, t]]$ is a complete Lie algebra with filtration defined by the grading given by the total degree in the parameters $s$ and $t$. In particular $\overline{\mathfrak{g}}_{1}=$ $s \mathfrak{g}[[s, t]]+t \mathfrak{g}[[s, t]]$. Thus by Theorem 4 , the map

$$
C_{-}: \mathfrak{g}^{-}[[s, t]]_{1} \times \mathfrak{g}^{+}[[s, t]]_{1} \rightarrow \mathfrak{g}[[s, t]]_{1}=\mathfrak{g}^{-}[[s, t]]_{1} \oplus \mathfrak{g}^{+}[[s, t]]_{1}
$$


is bijective with inverse

$$
D_{P}=(P, \tilde{P}) \circ \chi: \mathfrak{g}^{-}[[s, t]]_{1} \oplus \mathfrak{g}^{+}[[s, t]]_{1} \rightarrow \mathfrak{g}^{-}[[s, t]]_{1} \times \mathfrak{g}^{+}[[s, t]]_{1} .
$$

Then to prove items (2) and (3) in Theorem 20, and hence Theorem 18, we only need to show

Lemma 21. Let $U=s \mathfrak{g}^{-}[[s, t]] \times t \mathfrak{g}^{+}[[s, t]], V=s \mathfrak{g}^{-}[[s, t]] \oplus t \mathfrak{g}^{+}[[s, t]]$. Then $C_{-}$restricts to a bijective map from $U$ onto $V$.

Proof. The inclusion $C(U) \subset V$ is straightforward: if $a_{-} \in s \mathfrak{g}^{-}[[s, t]] \times t \mathfrak{g}^{+}[[s, t]]$ then clearly $a_{-}+a_{+} \in V$, and $\operatorname{BCH}\left(a_{-}, a_{+}\right) \in \operatorname{st} \mathfrak{g}[[s, t]]=s t \mathfrak{g}^{-}[[s, t]] \oplus \operatorname{st} \mathfrak{g}^{+}[[s, t]] \subseteq s \mathfrak{g}^{-}[[s, t]] \oplus t \mathfrak{g}^{+}[[s, t]]$.

Let us now prove the inclusion $\chi(V) \subseteq V$, i.e.,

$$
\chi\left(s \mathfrak{g}^{-}[[s, t]] \oplus t \mathfrak{g}^{+}[[s, t]]\right) \subseteq s \mathfrak{g}^{-}[[s, t]] \oplus t \mathfrak{g}^{+}[[s, t]],
$$

by using the definition of $B C H$-recursion: for any $v \in V, \chi(v)$ is the limit of $\chi_{(n)}(v)$ (for the topology defined by the filtration) recursively defined by $\chi_{(0)}(v)=v$ and

$$
\chi_{(n)}(v)=v-\operatorname{BCH}\left(P\left(\chi_{(n-1)}(v)\right), \tilde{P}\left(\chi_{(n-1)}(v)\right)\right) .
$$

It is clear, from the same argument as above, that $\chi_{(n-1)}(v) \in V$ implies $\chi_{(n)}(v) \in V$, so $\chi_{(n)}(v) \in V$ for any $n$ by induction. We then deduce $\chi(v) \in V$ by taking the limit, as $V$ is closed. We deduce immediately from this inclusion that $D_{P}(V) \subseteq U$, as $D_{P}(a)=$ $(P \circ \chi(a), \tilde{P} \circ \chi(a))$.

(2) Recall that the map $\Psi$ in Theorem 18.(2) is given by the following diagram

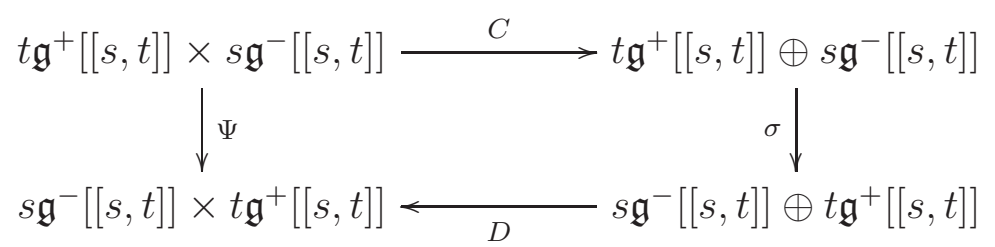

Here again $\sigma$ is just the identity map

$$
\sigma\left(t h^{+}+s h^{-}\right)=s h^{-}+t h^{+} .
$$

Then the proof of part (2) follows from part (1).

Part (3) is readily verified.

\section{Combinatorial Hopf algebras}

In Section 3 we applied the factorization property of the $B C H$-recursion $\chi$ together with the Rota-Baxter relation to Hopf algebras, in the context of the Hopf algebraic description of renormalization by Connes and Kreimer. This section consists of another application of $\chi$ to connected graded Hopf algebras. We analyze explicitly the even-odd decomposition of Aguiar, Bergeron, and Sottile $[\mathrm{ABS} 2003]^{2}$.

For a connected graded Hopf algebra $\left(\mathcal{H}=\oplus_{n \geq 0} \mathcal{H}_{n}, \Delta, m, \varepsilon, S\right)$, we define the grading operator $Y(h):=|h| h:=n h$, for a homogeneous element $h \in \mathcal{H}_{n}$, and extend linearly.

\footnotetext{
${ }^{2}$ We thank W. Schmitt for bringing the paper of Aguiar, Bergeron and Sottile [ABS2003] to our attention.
} 
The grading on $\mathcal{H}$ defines a canonical involutive automorphism on $\mathcal{H}$, denoted by $-: \mathcal{H} \rightarrow$ $\mathcal{H}, \bar{h}:=(-1)^{|h|} h=(-1)^{n} h$, for $h \in \mathcal{H}_{n}$. It induces by duality an involution on $\operatorname{Hom}(\mathcal{H}, \mathbb{K})$, $\bar{\phi}(h)=\phi(\bar{h})$ for $\phi \in \operatorname{Hom}(\mathcal{H}, \mathbb{K}), h \in \mathcal{H}$.

$\mathcal{H}$ naturally decomposes into $\mathcal{H}_{-}:=\bigoplus_{n>0} \mathcal{H}_{2 n-1}$ and $\mathcal{H}_{+}:=\bigoplus_{n \geq 0} \mathcal{H}_{2 n}$ on the level of vector spaces

$$
\mathcal{H}=\mathcal{H}_{-} \oplus \mathcal{H}_{+}
$$

with projectors $\pi_{ \pm}: \mathcal{H} \rightarrow \mathcal{H}_{ \pm}$. Such that for $h \in \mathcal{H}, \overline{\pi_{+}(h)}=\overline{h_{+}}=h_{+}$and $\overline{\pi_{-}(h)}=\overline{h_{-}}=-h_{-}$, $h=h_{-}+h_{+}$. As a remark we mention that $\mathcal{H}_{+}$is a subalgebra of $\mathcal{H}$, whereas $\mathcal{H}_{-}$is just a subspace, hence neither $\pi_{-}$nor $\pi_{+}:=\mathrm{id}_{\mathcal{H}}-\pi_{-}$are Rota-Baxter maps. Instead, we have $\mathcal{H}_{ \pm} \mathcal{H}_{ \pm} \subset \mathcal{H}_{+}$and $\mathcal{H}_{ \pm} \mathcal{H}_{\mp} \subset \mathcal{H}_{-}$.

The set of characters $G:=\operatorname{Char}(\mathcal{H}, \mathbb{K})$, i.e., multiplicative maps $\phi \in \operatorname{Hom}(\mathcal{H}, \mathbb{K})$, forms a group under convolution, defined by

$$
f \star g:=m_{\mathbb{K}} \circ(f \otimes g) \circ \Delta,
$$

for $f, g \in \operatorname{Hom}(\mathcal{H}, \mathbb{K})$. A character $\phi \in G$ is called even if it is a fixed point of the involution, $\bar{\phi}=\phi$, and is called odd if it is an anti-fixed point, $\bar{\phi}=\phi^{-1}=\phi \circ S$. The set of odd and even characters is denoted by $G_{-}, G_{+}$, respectively. Even characters form a subgroup in $G$. Whereas the set of odd characters forms a symmetric space. The following theorem is proved in [ABS2003].

Theorem 22. [ABS2003] Any $\phi \in \operatorname{Char}(\mathcal{H}, \mathbb{K})$ has a unique decomposition $\phi=\phi_{-} \star \phi_{+}$with $\phi_{-} \in G_{-}$being an odd character, and $\phi_{+} \in G_{+}$being an even character.

Both projectors $\pi_{-}: \mathcal{H} \rightarrow \mathcal{H}_{-}$and $\pi_{+}: \mathcal{H} \rightarrow \mathcal{H}_{+}$lift to $\operatorname{Hom}(\mathcal{H}, \mathbb{K})$. Implying for the complete filtered Lie algebra $\mathfrak{g}:=\partial \operatorname{Char}(\mathcal{H}, \mathbb{K})$, with filtration from $\mathcal{H}$, the direct decomposition $\mathfrak{g}=\mathfrak{g}_{-} \oplus \mathfrak{g}_{+}$into the Lie subalgebra $\mathfrak{g}_{+}$and the Lie triple system $\mathfrak{g}_{-}$. Such that for any $Z \in \mathfrak{g}$, we have $Z=Z_{-}+Z_{+}, Z_{ \pm} \in \mathfrak{g}_{ \pm}$unique. Then by Theorem 3, there is a $B C H$-recursion, $\chi: \mathfrak{g}_{1} \rightarrow \mathfrak{g}_{1}$ such that, for any $\phi=\exp (Z) \in \operatorname{Char}(\mathcal{H}, \mathbb{K}), Z \in \mathfrak{g}_{1}$, we have

$$
\phi=\exp (Z)=\exp \left(Z_{-}+Z_{+}\right)=\exp \left(\chi(Z)_{-}\right) \star \exp \left(\chi(Z)_{+}\right) .
$$

Here the exponential is defined with respect to the convolution product, $\exp (Z):=\sum_{n \geq 0} \frac{Z^{\star} n}{n !}$, but we will skip the $\star$ in the following to ease the notation.

Theorem 23. The even-odd factorization of a character in Theorem 22 coincides with the factorization in item (1) of Theorem 3.

The proof follows from the following properties of the involution $-: \mathcal{H} \rightarrow \mathcal{H}$. Recall the definition of an algebra involution on an algebra $A$, which is an algebra homomorphism $\jmath: A \rightarrow A$ such that $\jmath^{2}=\mathrm{id}_{A}$. Dually, define now a coalgebra involution to be a linear map $\jmath$ on a coalgebra $C$ such that $\jmath^{2}=\operatorname{id}_{C}$ and $(\jmath \otimes \jmath) \circ \Delta=\Delta \circ \jmath$.

Lemma 24. Let $\mathcal{H}$ be a connected filtered Hopf $\mathbb{K}$-algebra. Let $\jmath: \mathcal{H} \rightarrow \mathcal{H}$ be a coalgebra involution preserving the filtration. Then by pre-composition, J defines an algebra involution, still denoted by $\mathrm{J}$, on the filtered algebra $\mathcal{A}:=\operatorname{Hom}(\mathcal{H}, \mathbb{K})$ that preserves the filtration. 
Proof. For $h \in \mathcal{H}$, we have

$$
\begin{aligned}
\jmath(f \star g)(h)=(f \star g)(\jmath(h)) & =\left(m_{\mathbb{K}} \circ(f \otimes g) \circ \Delta \circ \jmath\right)(h) \\
& =\left(m_{\mathbb{K}} \circ(f \otimes g) \circ(\jmath \otimes \jmath) \circ \Delta\right)(h) \\
& =(\jmath(f) \star \jmath(g))(h) .
\end{aligned}
$$

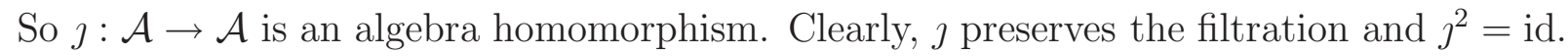

Lemma 25. Let $\mathrm{\jmath}$ be an algebra involution on the complete filtered algebra $\mathcal{A}$ that preserves the filtration.

(1) If $\jmath(a)= \pm a$ for $a \in \mathcal{A}_{1}$, then $\jmath(\exp (a))=\exp ( \pm a)$.

(2) Let $\mathcal{A}_{1, \pm}:=\left\{a \in \mathcal{A}_{1} \mid \jmath(a)= \pm a\right\}$ and $G_{ \pm}:=\left\{\eta \in 1+\mathcal{A}_{1} \mid \jmath(\eta)=\eta^{ \pm 1}\right\}$. Then $\exp \left(\mathcal{A}_{1,-}\right)=G_{-}$and $\exp \left(\mathcal{A}_{1,+}\right)=G_{+}$.

Proof. Since $\jmath$ preserves the filtration, $\mathcal{J}$ is a continuous map with respect to the topology defined by the filtration. So for any $a \in \mathcal{A}_{1}$, we have

$$
\jmath(\exp (a))=\jmath\left(\lim _{k \rightarrow \infty} \sum_{n=0}^{k} \frac{a^{n}}{n !}\right)=\lim _{k \rightarrow \infty} \jmath\left(\sum_{n=0}^{k} \frac{a^{n}}{n !}\right)=\lim _{k \rightarrow \infty} \sum_{n=0}^{k} \frac{\jmath(a)^{n}}{n !}=\exp (\jmath(a)) .
$$

Now item (1) of the lemma follows. Item (2) then follows from the bijectivity of exp.

Proof. (of Theorem 23) Now let $\phi \in \operatorname{Char}(\mathcal{H}, \mathbb{K})$, and $\jmath=^{-}$. Then by Lemma 24, we see that the induced $\jmath={ }^{-}$on $\operatorname{Hom}(\mathcal{H}, \mathbb{K})$ is an algebra involution that preserves the filtration. Then by Lemma 25.(1), $\exp \left(\chi(Z)_{-}\right)$(resp. $\left.\exp \left(\chi(Z)_{+}\right)\right)$is odd (resp. even). So Eq. (48) gives a decomposition of $\phi$ as an element of $G_{-}$and $G_{+}$. By Corollary 5 and Lemma 25.(2), we must have $\phi_{-}=\exp \left(\chi(Z)_{-}\right)$and $\phi_{+}=\exp \left(\chi(Z)_{+}\right)$, as needed.

We should remind the reader that the results of Proposition 8 do not apply here. We cannot calculate the exponentials $\phi_{ \pm}$using Spitzer's recursions in (18), since neither the projector $\pi_{+}$ nor $\pi_{-}$are of Rota-Baxter type. Nevertheless, the particular setting allows for a significant simplification of the $B C H$-recursion. In fact, using that $\phi_{ \pm} \in G_{ \pm}$hence $\overline{\phi_{-}}=\phi_{-}^{-1}$ and $\overline{\phi_{+}}=\phi_{+}$ and some algebra [MQZ2000], we find the following simple formula for $\pi_{-}(\chi(Z))=\chi(Z)_{-}$

$$
\pi_{-}(\chi(Z))=\pi_{-}(Z)+\frac{1}{2} \mathrm{BCH}\left(\pi_{-}(Z)+\pi_{+}(Z),-\pi_{-}(Z)+\pi_{+}(Z)\right) .
$$

This follows from Lemma 25 implying $\bar{\phi}=\jmath(\exp (Z))=\exp \left(-\pi_{-}(Z)+\pi_{+}(Z)\right)$ but also

$$
\jmath(\exp (Z))=\exp \left(-\chi(Z)_{-}\right) \star \exp \left(\chi(Z)_{+}\right) \text {. }
$$

Therefore, we have $\phi \star \bar{\phi}^{-1}=\phi_{-} \star \phi_{-}$which gives Eq. (49). From the factorization in Theorem 23 we derive a closed form for the $B C H$-recursion

$$
\chi(Z)=Z+\operatorname{BCH}\left(-\pi_{-}(Z)-\frac{1}{2} \operatorname{BCH}\left(Z, Z-2 \pi_{-}(Z)\right), Z\right) .
$$

We may remark that this gives an answer to Problem 19 in the particular setting just outlined, see also the remark after Theorem 20 . 


\section{Polar DeCOMposition}

The factorization of Aguiar et al. in the context of connected graded Hopf algebras is related to a general result elaborated in more detail in [MQZ2000, MQZ2001] and [Zan2004]. There it is shown that any connected Lie group $G$, together with an involutive automorphism $\sigma$ on $G$ allows locally for a decomposition similar to the above one.

We will briefly outline the setting of [MQZ2000, MQZ2001, Zan2004] and show that our $\mathrm{BCH}$-recursion provides an efficient mean for calculations. We should stress that the $\mathrm{BCH}$ recursion approach gives only formal series. Let $G$ be a connected Lie group, and $\mathfrak{g}$ its corresponding Lie algebra. We assume the existence of an involutive automorphism $\sigma$ on $G$. Let $G_{-}:=\left\{\psi \in G \mid \sigma(\psi)=\psi^{-1}\right\}$ denote the symmetric space of anti-fixed points of $\sigma$, and by $G_{+}:=\{\psi \in G \mid \sigma(\psi)=\psi\}$ we denote the subgroup of fixed points of $\sigma$. Also we denote by $\pi_{ \pm}$ the lifted projections on $\mathfrak{g}$ corresponding to $\sigma$. Hence for the Lie algebra $\mathfrak{g}$ we have the direct decomposition in terms of the images of these projectors, $\mathfrak{g}=\mathfrak{g}_{-} \oplus \mathfrak{g}_{+}$, where $\pi_{+}(\mathfrak{g})=$ : $\mathfrak{g}_{+}$is a Lie subalgebra and $\pi_{-}(\mathfrak{g})=: \mathfrak{g}_{-}$a Lie triple system.

In this setting Munthe-Kaas et al. derive a differentiable factorization of $\psi=\exp (t Z) \in G$, $Z \in \mathfrak{g}$ for sufficiently small parameter $t$. Using the additive decomposition of $\mathfrak{g}$ in terms of the projectors $\pi_{ \pm}$corresponding to $\sigma$, the factors, $\psi_{ \pm}(t):=\exp \left(X_{ \pm}(Z ; t)\right)$ in $\psi(t)=\psi_{-}(t) \psi_{+}(t)$ are calculated solving differential equations in $t$. This way explicit complicated recursions are derived for the terms in $X_{ \pm}(Z ; t)=\sum_{i>0} X_{ \pm}^{(i)}(Z) t^{i}$, using the relations between the spaces $\mathfrak{g}_{ \pm}$, i.e.,

$$
\left[\mathfrak{g}_{ \pm}, \mathfrak{g}_{\mp}\right] \subset \mathfrak{g}_{-}, \quad\left[\mathfrak{g}_{ \pm}, \mathfrak{g}_{ \pm}\right] \subset \mathfrak{g}_{+}
$$

The results coincide with those following form the simpler $B C H$-recursion map $\chi(2)$, which we state here again

$$
\chi(Z)=Z-\operatorname{BCH}\left(\pi_{-}(\chi(Z)), \pi_{+}(\chi(Z))\right),
$$

or Eq. (8) respectively its simple closed form in Eq. (50). The $\pi_{ \pm}$projections of the first three terms of $\chi(Z t)=t \sum_{k \geq 0} \chi^{(k)}(Z) t^{k}$ are $\pi_{ \pm}\left(\chi^{(0)}(Z)\right)=Z_{ \pm}$and for the next two non-trivial parts $(6,7)$ we find in order $t^{2}$

$$
\pi_{+}\left(\chi^{(1)}(Z)\right)=X_{+}^{(2)}=0 \quad \pi_{-}\left(\chi^{(1)}(Z)\right)=X_{-}^{(2)}=-\frac{1}{2}\left[Z_{-}, Z_{+}\right]
$$

and the even respectively odd projections in order $t^{3}$

$$
\begin{aligned}
\pi_{+}\left(\chi^{(2)}(Z)\right) & =X_{+}^{(3)}=\frac{1}{12}\left[Z_{-},\left[Z_{-}, Z_{+}\right]\right] \\
\pi_{-}\left(\chi^{(2)}(Z)\right) & =X_{-}^{(3)}=-\frac{1}{4}\left[Z_{+},\left[Z_{-}, Z_{+}\right]\right]+\frac{1}{12}\left[Z_{+},\left[Z_{-}, Z_{+}\right]\right] \\
& =-\frac{1}{6}\left[Z_{+},\left[Z_{-}, Z_{+}\right]\right] .
\end{aligned}
$$


The reader is invited to compare them with the results in Munthe-Kaas et al. [MQZ2000, MQZ2001] and especially Zanna's work [Zan2004] $]^{3}$. In our approach we work with one relatively simple $\mathrm{BCH}$ type recursion, $\chi(Z)$, respectively its closed form (50). Then we take the projections via $\pi_{ \pm}$to obtain $X_{ \pm}(Z ; t)$ up to third order in the parameter $t$. The parameter $t$ may be interpreted as providing us with the filtration (in the sense of formal power series). Hereby we use heavily the relations in (51). This seems to offer a simpler way for calculating the Lie algebra elements $X_{ \pm}(Z ; t) \in \mathfrak{g}_{ \pm}$. We only need higher expansion terms for $\chi$ and then project into $\mathfrak{g}_{ \pm}$. Relations (51) simplify the last step considerably.

Acknowledgements: The first author acknowledges greatly the support by the European Post-Doctoral Institute and Institut des Hautes Études Scientifiques (I.H.É.S.). He profited from discussions with M. Aguiar, J. M. Gracia-Bondía and D. Kreimer. Thanks goes to the Theory Department at the Physics Institute of Bonn University for warm hospitality. The second author thanks support from the NSF grant DMS-0505643 and Rutgers University Research Council, and thanks I.H.É.S. and Max Planck Institute for Mathematics in Bonn for hospitality. Many thanks go to J. Stasheff for comments and we appreciate helpful discussions with K. Barron, Y. Huang and J. Lepowsky. B. Fauser's useful remark is acknowledged. The third author greatly acknowledges constant support from the Centre National de la Recherche Scientifique (C.N.R.S.).

\section{REFERENCES}

[Agu2000] M. Aguiar, Prepoisson algebras, Lett. Math. Phys., 54, no. 4, 263-277, (2000).

[ABS2003] M. Aguiar, N. Bergeron and F. Sottile, Combinatorial Hopf algebras and generalized DehnSommerville relations, Composito Mathematica, 142, 1-30, 2006. [arXiv:math.CO/0310016]

[Atk1963] F. V. Atkinson, Some aspects of Baxter's functional equation, J. Math. Anal. Appl., 7, 1-30, (1963). [BBT2003] O. Babelon, D. Bernard and M. Talon, Introduction to classical integrable systems, Cambridge Monographs on Mathematical Physics. Cambridge University Press, Cambridge, (2003).

[BHL2000] K. Barron, Y. Huang and J. Lepowsky, Factorization of formal exponentials and uniformization, J. Algebra, 228, 551-579, (2000).

[Bax1960] G. Baxter, An analytic problem whose solution follows from a simple algebraic identity, Pacific J. Math., 10, 731-742, (1960).

[BelDri1982] A. A. Belavin and V. G. Drinfeld, Solutions of the classical Yang-Baxter equation for simple Lie algebras, Funct. Anal. Appl., 16, 159-180, (1982).

[BoPa1957] N. N. Bogoliubov and O. S. Parasiuk, On the multiplication of causal functions in the quantum theory of fields. Acta Math., 97, 227-266, (1957).

[Car1972] P. Cartier, On the structure of free Baxter algebras, Advances in Math., 9, 253-265, (1972).

[CK1999] A. Connes and D. Kreimer, Hopf algebras, Renormalization and Noncommutative Geometry, Comm. in Math. Phys., 199, 203-242, (1998). [arXiv:hep-th/9808042]

[CK2000] A. Connes and D. Kreimer, Renormalization in quantum field theory and the Riemann-Hilbert problem. I. The Hopf algebra structure of graphs and the main theorem, Comm. in Math. Phys., 210, no. 1, 249-273, (2000). [arXiv:hep-th/0003188]

\footnotetext{
${ }^{3}$ We would like to point to the recursive equation (1.1) on page 2 for the $X_{-}^{(k)}=: X_{k}$, and equation (3.5) on page 7 for $X_{+}^{(l)}=: Y_{l}$ in [Zan2004]
} 
[CK2001] A. Connes and D. Kreimer, Renormalization in quantum field theory and the Riemann-Hilbert problem. II. The $\beta$-function, diffeomorphisms and the renormalization group, Comm. in Math. Phys., 216, 215-241, (2001). [arXiv:hep-th/0003188]

[Col1984] J. C. Collins, Renormalization, Cambridge Monographs on Mathematical Physics, Cambridge University Press, Cambridge, (1984).

[EGK2004] K. Ebrahimi-Fard, L. Guo and D. Kreimer, Spitzer's Identity and the Algebraic Birkhoff Decomposition in pQFT, J. Phys. A: Math. Gen., 37, 11037-11052, (2004). [arXiv:hep-th/0407082]

[EGK2005] K. Ebrahimi-Fard, L. Guo and D. Kreimer, Integrable Renormalization II: the General case, Ann. H. Poincaré, 6, 369-395, (2005). [arXiv:hep-th/0403118]

[EGGV2006] K. Ebrahimi-Fard, J. M. Gracia-Bondía, L. Guo and J.C. Várilly, Combinatorics of renormalization as matrix calculus, Phys. Lett. B., 632, no 4, 552-558, (2006). [arXiv:hep-th/0508154]

[EG2005] K. Ebrahimi-Fard and L. Guo, Matrix Representation of Renormalization in Perturbative Quantum Field Theory, submitted, preprint: August 2005 arXiv:hep-th/0508155.

[EK2005] K. Ebrahimi-Fard and D. Kreimer, Hopf algebra approach to Feynman diagram calculations, J. Phys. A: Math. Gen., 38, R385-R406, 2005. [arXiv:hep-th/0510202]

[FGB2005] H. Figueroa and J. M. Gracia-Bondía, Combinatorial Hopf algebras in quantum field theory I, Reviews of Mathematical Physics, 17, 881-976, (2005). [arXiv:hep-th/0408145]

[Go1982] R. Godement, Introduction à la théorie des groupes de Lie, Reprint of the 1982 original. SpringerVerlag, Berlin (2004).

[Hepp1966] K. Hepp, Proof of the Bogoliubov-Parasiuk theorem on renormalization, Comm. in Math. Phys., 2, 301-326, (1966).

[Krei1998] D. Kreimer, On the Hopf algebra structure of perturbative quantum field theories, Adv. Theor. Math. Phys., 2, 303-334, (1998). [arXiv:q-alg/9707029]

[Krei1999] D. Kreimer, Chen's iterated integral represents the operator product expansion, Adv. Theor. Math. Phys., 3, no. 3, 627-670, (1999). [arXiv:hep-th/9901099]

[Krei2002] D. Kreimer, Combinatorics of (perturbative) Quantum Field Theory, Phys. Rep., 363, 387-424, (2002). [arXiv:hep-th/00110059]

[King1962] J. F. C. Kingman, Spitzer's identity and its use in probability theory, J. London Math. Soc., 37, 309-316, (1962).

[Lod1994] J.-L. Loday, Série de Hausdorff, idempotents Eulériens et algèbres de Hopf, Expo. Math., 12, 165$178,(1994)$.

[Mag1954] W. Magnus, On the exponential solution of differential equations for a linear operator, Comm. Pure Appl. Math., 7, 649-673, (1954).

[Man2001] D. Manchon, Hopf algebras, from basics to applications to renormalization, Comptes-rendus des Rencontres mathématiques de Glanon 2001. [arXiv:math.QA/0408405]

[MQZ2000] H. Z. Munthe-Kaas, G. R. W. Quispel and A. Zanna, The polar decomposition of Lie groups with involutive automorphisms, Technical Report, no. 191, Dept. of Informatics, Univ. of Bergen, Norway, (2000).

[MQZ2001] H. Z. Munthe-Kaas, G. R. W. Quispel and A. Zanna, Generalized polar decompositions on Lie groups with involutive automorphisms, Found. Comput. Math.,1, no. 3, 297-324, (2001).

[Poli2002] A. Polishchuk, Classical Yang-Baxter equation and the $A_{\infty}$-constraint, Adv. Math., 168, no. 1, $56-95,(2002)$.

[Reu1993] C. Reutenauer, Free Lie Algebras, Oxford University Press, Oxford, 1993.

[Rota1969] G.-C. Rota, Baxter algebras and combinatorial identities. I, II., Bull. Amer. Math. Soc., 75, 325329, (1969); ibid. 75, 330-334, (1969).

[RoSm1972] G.-C. Rota and D. Smith, Fluctuation theory and Baxter algebras, Istituto Nazionale di Alta Matematica, IX, 179, (1972). Reprinted in: "Gian-Carlo Rota on Combinatorics: Introductory 
papers and commentaries", J.P.S. Kung Ed., Contemp. Mathematicians, Birkhäuser Boston, Boston, MA, 1995.

[Rota1995] G.-C. Rota, Baxter operators, an introduction, In: "Gian-Carlo Rota on Combinatorics, Introductory papers and commentaries", J.P.S. Kung Ed., Contemp. Mathematicians, Birkhäuser Boston, Boston, MA, 1995.

[Rota1998] G.-C. Rota, Ten mathematics problems I will never solve, Invited address at the joint meeting of the American Mathematical Society and the Mexican Mathematical Society, Oaxaca, Mexico, December 6, 1997. DMV Mittellungen, Heft 2, 45-52, (1998).

[Sem1983] M. A. Semenov-Tian-Shansky, What is a classical r-matrix?, Funct. Ana. Appl., 17, no.4, 254-272, (1983).

[Sem2000] M. A. Semenov-Tian-Shansky, Integrable Systems and Factorization Problems, Lectures given at the "Faro International Summer School on Factorization and Integrable Systems" (Sept. 2000), Birkhäuser 2003. [arXiv:nlin.SI/0209057]

[Spit1956] F. Spitzer, A combinatorial lemma and its application to probability theory, Trans. Amer. Math. Soc., 82, 323-339, (1956).

[Var1984] V. S. Varadarajan, Lie Groups, Lie Algebras, and Their Representations, Springer-Verlag, (1984).

[Wen1962] J. G. Wendel, A brief proof of a theorem of Baxter, Math. Scand., 11, 107-108, (1962).

[Zan2004] A. Zanna, Recurrence relations and convergence theory of the generalized polar decomposition on Lie groups, Math. Comp., 73, no. 246, 761-776, (2004).

[Zim1969] W. Zimmermann, Convergence of Bogoliubov's method of renormalization in momentum space, Comm. in Math. Phys., 15, 208-234, (1969).

I.H.É.S., Le Bois-Marie, 35, Route de Chartres, F-91440 Bures-Sur-Yvette, France

E-mail address: kurusch@ihes.fr

$U R L:$ http://www.th.physik. uni-bonn.de/th/People/fard/

Department of Mathematics and Computer Science, Rutgers University, Newark, NJ 07102, U.S.A.

E-mail address: liguo@newark.rutgers.edu

$U R L:$ http://newark.rutgers.edu/ liguo/

Université Blaise Pascal, C.N.R.S.-UMR 6620, 63177 Aubière, France

E-mail address: manchon@math.univ-bpclermont.fr

$U R L$ : http://math.univ-bpclermont.fr/ manchon/ 